Nee Pang: None declared, Darren Tay: None declared, Ying Ying Leung Grant/research support from: Abbvie, Novartis, Speakers bureau: Abbvie and Novartis, Speakers bureau: Novartis DOI: 10.1136/annrheumdis-2019-eular.2599

\section{THU0446 DIACEREIN PROVEN AS EFFECTIVE AS CELECOXIB IN REDUCING PAIN AND SYMPTOMS IN KNEE OSTEOARTHRITIS PATIENTS IN A MULTICENTRE DOUBLE-BLIND RANDOMISED STUDY (DISSCO)}

Jean-Pierre Pelletier ${ }^{1}$, Jean-Pierre Raynauld ${ }^{2}$, Patrice Paiement ${ }^{3}$, Johanne MartelPelletier ${ }^{1}{ }^{1}$ Osteoarthritis Research Unit, University of Montreal Hospital Research Centre (CRCHUM), Montreal, Canada; ${ }^{2}$ Institut de Rhumatologie de Montréal, Montreal, Canada; ${ }^{3}$ ArthroLab Inc., Montreal, Canada

Background: There was a need to ascertain in a clinical trial the comparative efficacy of diacerein, an IL-1 inhibitor, vis-à-vis celecoxib, a COX-2 inhibitor, in relieving knee osteoarthritis (OA) symptoms.

Objectives: The primary outcome of this study was to show that diacerein is non-inferior to celecoxib in terms of pain reduction (WOMAC A pain subscale) after 6 months of treatment in moderate-to-severe symptomatic knee OA patients.

Methods: A randomised double-blind multicentre trial was conducted in four European countries (Spain, Belgium, Austria, Czech Republic) and in Canada evaluating treatment with diacerein versus celecoxib in patients with $\mathrm{OA}$ diagnosed according to ACR criteria, with $\mathrm{KL}$ grade 2-3 knee OA and moderate-tosevere pain (VAS pain score [0-100 mm] while walking on a flat surface $>40$ $\mathrm{mm}$ ). Eligible patients were randomised to treatment for 6 months with either diacerein $50 \mathrm{mg}$ once daily for the first month and twice daily thereafter, or celecoxib $200 \mathrm{mg}$ once daily. The primary outcome was the change from baseline in WOMAC pain subscale (0-50 scale) after 6 months of treatment. Secondary outcomes included WOMAC function and stiffness, VAS pain, presence of joint swelling/effusion, rescue medication consumption, percentage of OMERACT-OARSI responders, and SF-36. A total of 380 patients were randomised in the study. The primary outcome assessment on the per protocol set (PPS) $(n=288)$ was followed by sensitivity analysis on the ITT population $(n=370)$. Exploratory statistical analysis on other efficacy criteria and safety were performed.

Results: The analysis of the PPS population showed that the adjusted mean change in WOMAC pain was -11.14 (SEM, 0.91) with diacerein $(n=140)$ and $-11.82(0.89)$ with celecoxib $(n=148)$. Diacerein met the noninferiority criterion, as the upper bound of the $95 \% \mathrm{Cl}$ is inferior to the pre-specified non-inferiority margin of 5 (inter-group difference $0.67 ; 95 \%$ $\mathrm{Cl}-1.83$ to $3.18 ; \mathrm{p}=0.597$ ). The sensitivity analysis using the ITT population was supportive of those results. All other exploratory outcomes demonstrated no difference between the two treatment groups. The OMERACT-OARSI responder rate (PPS) at 6 months was similar in the diacerein $(62.1 \%)$ and celecoxib $(60.1 \%)$ groups. The incidence of adverse events related to drug treatment was in general low and balanced between groups. The only exception was a greater incidence of gastrointestinal side effects (diarrhoea) in the diacerein vs. the celecoxib group ( $10.2 \%$ vs. $3.7 \%$, respectively), but accounted for $4.8 \%$ of permanent discontinuation in the diacerein group compared to $1.6 \%$ in the celecoxib group. In all but one patient, this diarrhoea was considered to be of a mild-to-moderate grade with complete resolution in all cases.

Conclusion: This clinical trial showed that in patients with moderate-tosevere knee $O A$ pain, diacerein has comparable efficacy to celecoxib with respect to reducing pain and stiffness and improving function after 6 months in a clinically relevant manner. The drug also demonstrated a good safety profile with positive benefit to risk ratio.

Disclosure of Interests: : Jean-Pierre Pelletier Shareholder of: Shareholder in ArthroLab Inc., Grant/research support from: Study funded by TRB Chemedica SA, Consultant for: TRB Chemedica SA, Jean-Pierre Raynauld Consultant for: ArthroLab Inc., Patrice Paiement Employee of: ArthroLab Inc., Johanne Martel-Pelletier Shareholder of: ArthroLab Inc., Consultant for: TRB Chemedica SA

DOI: 10.1136/annrheumdis-2019-eular.899

\section{THU0447 THE RELATIVE EFFICACY OF TOPICAL NON- STEROIDAL ANTI-INFLAMMATORY DRUGS AND CAPSAICIN IN OSTEOARTHRITIS: MOVING FROM AVERAGE TREATMENT EFFECTS TO INDIVIDUAL TREATMENT PREFERENCES}

Monica Persson ${ }^{1}$, Joanne Stocks ${ }^{1}$, Aliya Sarmanova ${ }^{2}$, Gwen Fernandes ${ }^{2}$, Gyula Varadi ${ }^{3}$, Mohammad Hashem Hashempur ${ }^{4}$, Robbert van Haselen ${ }^{5}$, David Walsh ${ }^{1}$, Michael Doherty ${ }^{1}$, Weiya Zhang ${ }^{1} .{ }^{1}$ University of Nottingham, Nottingham, United Kingdom; ${ }^{2}$ University of Bristol, Bristol, United Kingdom; ${ }^{3}$ BioPhysics Pharma, Inc., Beverly, United States of America; ${ }^{4}$ Fasa University of Medical Sciences, Fasa, Iran (Islamic Republic of); International Institute for Integrated Medicine, London, United Kingdom

Background: Pain is an important issue for people with osteoarthritis (OA). Topical non-steroidal anti-inflammatory drugs (tNSAIDs) and capsaicin are recommended treatments, but reduce $O A$ pain by different mechanisms.

Objectives: To [1] determine the relative efficacy of tNSAIDs and capsaicin in $O A$ and [2] identify predictors of response that may allow tailoring of topical therapy to the individual.

Methods: Systematic literature searches were conducted up to 16/11/2015 for randomised controlled trials (RCTs) of tNSAIDs or capsaicin in OA. Placebo-controlled RCTs were pooled in conventional meta-analyses (CMA) for specific (difference between treatment and placebo) and overall (pain reduction in treatment arm) treatment effects. Bayesian network meta-analysis (NMA) compared treatments via placebo. RCT data custodians were contacted and an individual patient data meta-analysis (IPDMA) examined for predictors of response.

A pilot $n$-of-1 trial series (PNTS) examined treatment effects, patient preferences, and predictors of response. Participants with painful radiographic knee OA were allocated to three treatment cycles, each comprising four weeks treatment with $5 \%$ ibuprofen gel and $0.025 \%$ capsaicin cream in a random order.

Effect sizes (ES) are Hedges' $\mathrm{g}$ and 95\% confidence interval (Cl) or credible interval $(\mathrm{Crl})$. Predictors of response were identified in regression modelling for treatment-by-covariate interactions (specific effect) or covariate associations (overall effect). 9 and 25 predictors were examined in IPDMA and PNTS, respectively.

Results: Of 63 tNSAID and 10 capsaicin RCTs identified, CMA and NMA analysed 21 tNSAID ( $n=6191)$ and five capsaicin $(n=415)$ RCTs. The quality of evidence was moderate for tNSAIDs and very low for capsaicin. Overall treatment effects were large and likely clinically significant (tNSAIDs ES $1.23,95 \% \mathrm{Cl} 1.06-1.41$; capsaicin ES $1.05,95 \% \mathrm{Cl} 0.52$ 1.57). tNSAIDs (all drugs/doses grouped) were superior to placebo (ES $0.31,95 \% \mathrm{Cl} 0.20-0.41$ ), but capsaicin was only effective at $0.025 \%$ concentration (ES $0.41,95 \% \mathrm{Cl} 0.17-0.64$; ES of all capsaicin RCTs 0.28 , $95 \% \mathrm{Cl}-0.04-0.60)$. On average, tNSAIDs and capsaicin were equally effective (ES $0.04,95 \% \mathrm{Crl}-0.29-0.37$ ).

IPD were provided for $15(n=3889)$ tNSAID RCTs, including $11 \quad(n=3140)$ placebo-controlled RCTs. No IPD were obtained for capsaicin. A significant but small interaction was observed between sex and tNSAID use for pain relief, with women reporting greater effect (11 RCTs, $n=2939$, $\mathrm{p}=0.023$ ).

22 participants enrolled in the PNTS and completed 104 treatment periods. Clinically important pain relief $(\geq 1$ on $0-10$ numeric rating scale) was achieved in $64 \%$ of periods, with no difference in efficacy between treatments overall $(p=0.271)$. Individual responses to treatment varied, with c.60\% of people preferring one treatment over the other, but without clear predictors for the preference.

Conclusion: tNSAIDs and capsaicin may relieve OA pain, but treatment effects from group comparisons do not directly translate to treatment out comes at an individual level. No baseline characteristics robustly determine which treatment is more likely to benefit an individual. Patients may benefit from trying both treatments to determine which is better for them. Acknowledgement: M Underwood (Warwick University), A Suter (Bioforce AG/A.Vogel Produkte), GR Mautone (IBSA Institut Biochimique SA), and GSK for sharing IPD; D Burke for statistical advice; and D McWilliams for trial randomisation

Disclosure of Interests: Monica Persson: None declared, Joanne Stocks Grant/research support from: Co-I on research grant from Pfizer/Eli Lilly, Aliya Sarmanova: None declared, Gwen Fernandes: None declared, Gyula Varadi Employee of: Inpellis, Inc. (previous); BioPhysics Pharma, Inc (current), Mohammad Hashem Hashempur: None declared, Robbert van Haselen Consultant for: Consultancy services for homeopathic pharmaceutical companies, David Walsh Consultant for: Pfizer, GlaxoSmithKline. Consultancies on arthritis pain, Speakers bureau: Pfizer Ltd for a talk at OARSI 2018, Michael Doherty Grant/research support from: AstraZeneca funded the Nottingham Sons of Gout study, Consultant for: Advisory Boards on Grunenthal and Mallinckrodt, Weiya Zhang Consultant for: 
Grunenthal for advice on gout management, Speakers bureau: Bioiberica as an invited speaker for EULAR 2016 satellite symposium DOI: 10.1136/annrheumdis-2019-eular.699

\section{\begin{tabular}{|l|l}
\hline THU0448 DISEASE BURDEN IN OSTEOARTHRITIS (OA) IS \\
\hline
\end{tabular} SIMILAR TO RHEUMATOID ARTHRITIS (RA) FROM THE PATIENT'S PERSPECTIVE, SLIGHTLY HIGHER IN RA AT PRESENTATION, SIMILAR ONE YEAR LATER, AND SLIGHTLY HIGHER IN OA TWO YEARS LATER AT ONE PRIVATE PRACTICE SETTING}

Martin Bergman ${ }^{1}$, Mariam Riad ${ }^{2}$, Theodore Pincus ${ }^{2} .{ }^{1}$ Drexel University College of Medicine, Philadelphia, United States of America; ${ }^{2}$ Rush University Medical Center, Chicago, United States of America

Background: Disease burden traditionally has been regarded as considerably greater in rheumatoid arthritis (RA) versus osteoarthritis (OA). However, recent reports of cross-sectional data indicate similar disease burden in OA vs RA according to MDHAQ/RAPID3 (multidimensional health assessment questionnaire/routine assessment of patient index data). One concern is that these findings may reflect only better treatment for RA, and initial disease burden may be considerably higher in RA vs OA.

Objectives: To analyze disease burden in patients with $O A$ vs RA at baseline and 1- and 2-year follow-up according to MDHAQ/RAPID3 scores in routine care at a single rheumatologist private practice site. Methods: All patients seen in routine care at this site complete an MDHAQ at each visit in the waiting area prior to seeing the rheumatologist. The MDHAQ includes three 0-10 scores for physical function, pain visual analogue scale (VAS), and patient global VAS, compiled into a 030 RAPID3, as well as 0-10 fatigue VAS, and 0-48 self-report painful joint count scores. Mean MDHAQ scores were compared in OA versus RA patients at 1st visit and visits 1 and 2 years later, using $t$ tests, adjusted for age and body mass index (BMI) using analysis of variance (ANOVA).

Results: Among $101 \mathrm{OA}$ and 175 patients with RA, at first visit, all MDHAQ scores except pain VAS were statistically significantly higher in RA vs OA, e.g., mean 0-30 RAPID3 was 11.9 in OA and 13.7 in RA. However, none of these differences appear clinically significant (Table). After 1year, all scores were improved, but more in RA vs OA patients (Table), e. g., mean RAPID3 of 11.5 in OA and 10.9 in RA; no differences between $\mathrm{OA}$ and RA were statistically or clinically significant. After 2-years, mean RAPID3 was 11.9 in OA vs 9.0 in RA, indicating continued improvement in RA but little change in OA. All scores other than fatigue VAS were higher in $O A$, including the self-report painful joint count. Differences between $\mathrm{OA}$ and $\mathrm{RA}$ were explained only minimally by age and BMI.

Table. Mean MDHAQ scores in patients with primary OA or RA at 1st visit, and 1 or 2 years later

\begin{tabular}{|c|c|c|c||c|c|c||c|c|c||c||c|}
\hline Mean scores at... & \multicolumn{3}{|c||}{ 1st visit } & \multicolumn{3}{|c||}{ 1-year later } & \multicolumn{3}{|||}{ 2-years later } & \multicolumn{2}{|c|}{ PChange in OAvsRA } \\
\hline Variable: & OA & RA & P & OA & RA & P & OA & RA & P & 1 year & 2 years \\
\hline \# patients & 101 & 175 & & 101 & 175 & & 45 & 113 & & & \\
\hline RAPID3 (0-30) & 11.9 & 13.7 & 0.03 & 11.5 & 10.9 & 0.50 & 11.9 & 9.0 & 0.02 & 0.004 & 0.0004 \\
\hline Function (0-10) & 0.63 & 0.78 & 0.03 & 0.61 & 0.66 & 0.43 & 0.72 & 0.53 & 0.046 & 0.119 & 0.009 \\
\hline Pain (0-10) & 5.0 & 5.5 & 0.16 & 4.9 & 4.3 & 0.43 & 4.7 & 3.6 & 0.03 & 0.004 & 0.004 \\
\hline PATGL (0-10) & 4.8 & 5.6 & 0.01 & 4.6 & 4.4 & 0.54 & 4.9 & 3.7 & 0.009 & 0.005 & 0.0001 \\
\hline Fatigue (0-10) & 3.9 & 4.9 & 0.008 & 3.7 & 4.0 & 0.43 & 3.6 & 4.0 & 0.41 & 0.073 & 0.359 \\
\hline $\begin{array}{c}\text { Self-report painful } \\
\text { JC 48 (0-48) }\end{array}$ & 12.0 & 15.4 & 0.014 & 9.5 & 11.7 & 0.10 & 11.9 & 10.2 & 0.35 & 0.415 & 0.035 \\
\hline
\end{tabular}

$O A=$ osteoarthritis, $R A=$ rheumatoid arthritis, $R A P I D 3=$ routine assessment of patient index

data, PATGL= patient global assessment, JC=joint count

Conclusion: Most MDHAQ/RAPID3 scores were higher in RA than in OA at the first visit, indicating greater severity of RA, although OA was almost as severe. One year later, scores were similar with no statistically significant differences. Two years later, most scores were higher in OA. These findings likely reflect superior treatments for RA vs OA. At an individual level, patients with primary OA may have better or poorer status than other patients with primary RA. Nonetheless, at a group level, the severity of disease burden in OA appears almost as great as in RA, and becomes greater over the next 2 years, likely as a result of better treatment. The severity of $\mathrm{OA}$ is underrated, suggesting a need for increasing resources for research toward better treatment for OA.

Disclosure of Interests: Martin Bergman Shareholder of: Johnson and Johnson (parent company of Janssen), Consultant for: AbbVie, Amgen, BMS, Celgene, Genentech/Roche, Janssen, Merck, Novartis, Pfizer, and Sanofi/Regeneron, Speakers bureau: AbbVie, Amgen, BMS, Celgene,
Genentech/Roche, Janssen, Merck, Novartis, Pfizer, and Sanofi/Regeneron, Mariam Riad: None declared, Theodore Pincus: None declared DOI: 10.1136/annrheumdis-2019-eular.5883

\section{THU0449 EFFECT MODERATION OF ANALGESIC TREATMENT OUTCOMES BY DEPRESSION IN PERSONS WITH OR AT-RISK FOR SYMPTOMATIC KNEE OSTEOARTHRITIS}

Alan Rathbun ${ }^{1}$, Michelle Shardell ${ }^{2}$, Joseph Gallo ${ }^{3}$, Alice Ryan ${ }^{1}$, Elizabeth Stuart ${ }^{3}$, Megan Schuler ${ }^{4}$, Michelle Yau ${ }^{5}$, Marc Hochberg ${ }^{1} .{ }^{1}$ University of Maryland Schoo of Medicine, Baltimore, United States of America; ${ }^{2}$ National Institute on Aging, Baltimore, United States of America; ${ }^{3}$ Johns Hopkins Bloomberg School of Public Health, Baltimore, United States of America; ${ }^{4}$ RAND Corportation, Boston, United States of America; ${ }^{5} \mathrm{Hinda}$ and Arthur Marcus Institute for Aging Research, Boston, United States of America

Background: Depression often accompanies knee osteoarthritis (OA), exacerbates pain severity, and may negatively affect analgesic treatment outcomes.

Objectives: To determine whether depression moderates the effect of analgesics on pain severity in persons with or at-risk for symptomatic knee OA. Methods: Participants $(n=2059)$ were from the Osteoarthritis Initiative, with or at risk for symptomatic knee $\mathrm{OA}$, and had complete data on selected measures at baseline and four annual follow-up visits. Analgesic initiation (acetaminophen, non-steroidal anti-inflammatory drugs, opioids) was assessed at three annual follow-up visits in those who were not analgesic users at baseline. Depression was evaluated concurrent to assessment of analgesic use with the Center for Epidemiological Studies Depression (CES-D) scale using the corresponding CES-D screening threshold (CES-D score $\geq 16$ ). Pain severity at the fourth annual followup visit was the outcome and measured with the Western Ontario and McMaster Universities Osteoarthritis Index (WOMAC) pain scale (rescaled range $=0-100$ ). Time-invariant confounders measured at baseline were: age, gender, race, education, marital status, employment, health insurance, smoking, alcohol consumption, Charlson comorbidity index, and symptomatic knee OA status. Time-varying confounders measured at three annual follow-up visits were: WOMAC pain score, Kellgren-Lawrence grade, body mass index, physical performance, knee injuries, and knee injections. Structural nested mean models appropriate for evaluating time-varying effect moderation of dynamic treatments by evolving effect modifiers were implemented using an inverse-probability-of-treatment-weighting regression-with-residuals approach.

Results: In non-depressed participants, analgesic treatment effect at years one, two, and three on pain severity at year four was minimal (Figure 1). Time-specific associations in the non-depressed ranged from -1.18 (95\% Cl: $-2.94,0.59)$ to $0.81(95 \% \mathrm{Cl}:-0.99,2.61)$ and were not statistically significant. The persistent use of analgesics at years one and two $(\beta=-2.17 ; 95 \% \mathrm{Cl}:-4.62,0.27)$ or years one, two, and three $(\beta=-$ $1.36 ; 95 \% \mathrm{Cl}:-4.22,1.50)$ did not increase the magnitude of the treatment effect in non-depressed participants. In contrast, time-specific associations in depressed participants increased during follow-up from 0.61 (95\% Cl: $-9.17,10.38)$ to $-9.64(95 \% \mathrm{Cl}:-17.96,-1.33)$, and the treat ment effect of year three analgesic use on year four pain severity was statistically significant. The magnitude of the treatment effect increased from year one with persistent analgesic use at years one and two $(\beta=$ $-5.75 ; 95 \% \mathrm{Cl}:-20.65,9.15)$ and years one, two, and three $(\beta=-15.39$; $95 \% \mathrm{Cl}:-33.99,3.21)$. However, effect moderation was only significant concerning year three analgesic use, where the subsequent difference in treatment effect between depressed and non-depressed participants was 10.46 (95\% Cl: $-18.97,-1.94)$.

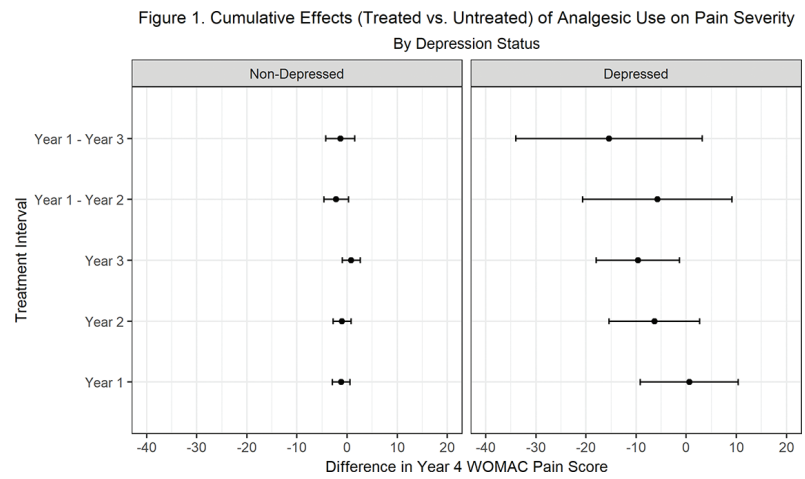

Abstract THU0449 - Figure 1 